\title{
Evaluation of A Resilience Embedded System Using Probabilistic Model-Checking
}

\author{
Ling Fang, Yoriyuki Yamagata, Yutaka Oiwa \\ National Institute of Advanced Industrial Science and Technology, Japan \\ \{fang-ling, yoriyuki.yamagata, y.oiwa\}@aist.go.jp
}

\begin{abstract}
If a Micro Processor Unit (MPU) receives an external electric signal as noise, the system function will freeze or malfunction easily. A new resilience strategy is implemented in order to reset the MPU automatically and stop the MPU from freezing or malfunctioning. The technique is useful for embedded systems which work in non-human environments. However, evaluating resilience strategies is difficult because their effectiveness depends on numerous, complex, interacting factors.

In this paper, we use probabilistic model checking to evaluate the embedded systems installed with the above mentioned new resilience strategy. Qualitative evaluations are implemented with 6 PCTL formulas, and quantitative evaluations use two kinds of evaluation. One is system failure reduction, and the other is ADT (Average Down Time), the industry standard. Our work demonstrates the benefits brought by the resilience strategy. Experimental results indicate that our evaluation is cost-effective and reliable.
\end{abstract}

\section{Introduction}

If a Micro Processor Unit (MPU) receives an external electric signal as noise, the system function will freeze or malfunction easily [1]. Therefore, a resilience strategy for the MPU in a non-human environment is needed so that the embedded system can continue working [2]. This paper considers a new resilience technique in order to reset the MPU automatically and to prevent the MPU from freezing. The application programs restart from backup data. Therefore, the temporal failure has no apparent effect on the application programs. This resilience strategy is implemented as a system that works between the hardware and operating system. This system is named FUJIMI 1 . In the FUJIMI system, Non-Maskable Interrupts (NMI) and Reset signals (RST) are triggered [3] periodically to save data for backup and then the backup data is used to reset the MPU. Figure 1 is an example comparing two embedded systems with and without the FUJIMI system installed to show that system failure is reduced from once a day to once every 4 days.

Evaluating such resilience strategies is a challenging task because the evaluations depend on many complex factors including probabilistic noise environments. As a result, empirical experimentation is often a more useful approach for evaluation than proofs or formal approaches. However, evaluating a live system under noise attack is time-intensive and costly, and only achieves results under limited cases. This paper presents a formal approach to providing cost-effective and reliable evaluations of resilience strategies by means of simulation and probabilistic model checking. We simulate the resilience strategy with a probabilistic model and present a qualitative verification and quantitative assessment for the model. We use PRISM [4] [5], which is a tool for formal modeling and analysis of systems that exhibit probabilistic behavior. DTMC (Discrete-Time Markov Chain) is the specification language, and PCTL (Probabilistic Computation Tree Logic) is used for analysis [6].

\footnotetext{
${ }^{1}$ FUJIMI means always alive in Japanese.
}

J. Pang and Y. Liu (Eds.): 3rd International Workshop on Engineering Safety and Security Systems 2014 (ESSS 2014)

EPTCS 150, 2014, pp. 3549 doi 10.4204/EPTCS.150.4 (c) Ling Fang, Yoriyuki Yamagata, Yutaka Oiwa

This work is licensed under the Creative Commons Attribution License. 

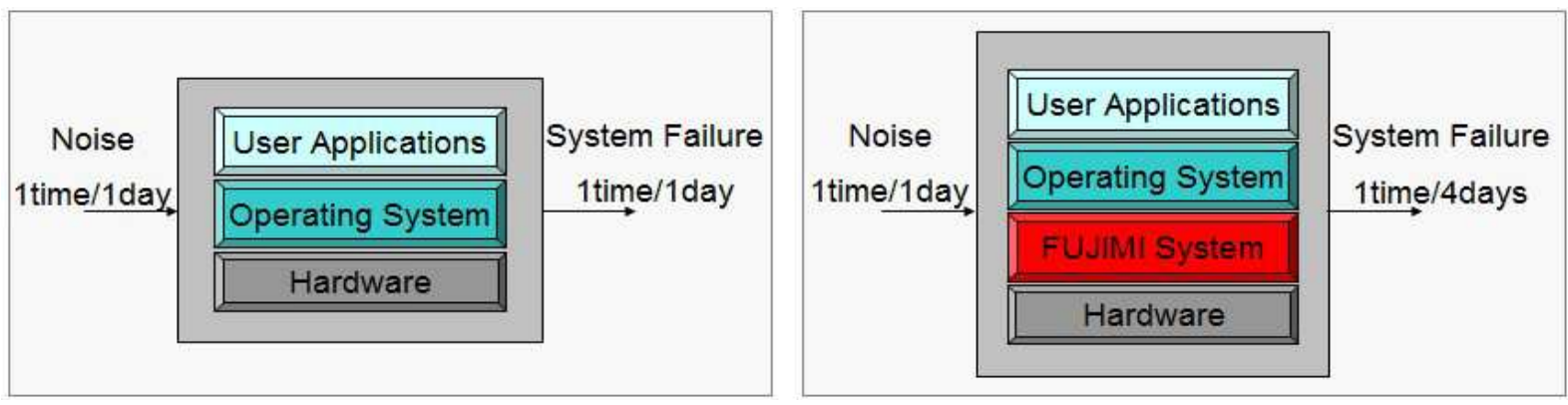

Figure 1: The Embedded System With (right) And Without (left) The FUJIMI system

The results of qualitative verification support certain claims and expectations of resilience strategy. Quantitative assessments provide two types of evidence to demonstrate how the resilience strategy improves the system effectiveness: reduction of system failure and standard industry criteria ADT. In other words, the qualitative verification ensures the analysis results from the model are correct and the quantitative assessments demonstrate the benefits of the resilience strategy. Moreover, the system can be optimized for different noise environments and strategy variations. The evaluations were difficult to acquire when testing with a fully implemented embedded system until our work.

The remainder of this paper is structured as follows: Section 2 is a preliminary explanation for probabilistic model checking and PCTL. Section 3 explains the principle and the resilience strategy of the FUJIMI system. Section 4 discusses how to construct the formal probabilistic model. Section 5 describes our methods for system evaluation. Section 6 reviews several examples of related work. In Section 7, the paper concludes with a discussion about our contributions and future research.

\section{Probabilistic model checking and PCTL}

Probabilistic model checking is a formal verification technique for establishing the correctness, performance, and reliability of systems which exhibit stochastic and probabilistic behavior. As in conventional model checking, a model of the probabilistic system, usually some variant of a Markov chain, is built and then subjected to algorithmic analysis to establish whether it satisfies a given specification. The specifications are usually stated as formula of probabilistic temporal logic which, in addition to conventional modalities, may include probabilistic operators whose outcome is true/false depending on the probability of certain executions.

In this paper, we report on our experiences using the probabilistic model checker PRISM [4] [5]. We use DTMC as the modeling language and use PTCL to verify the properties [6].

\subsection{DTMC (Discrete-Time Markov Chains) Model}

DTMCs are defined as state-transition systems augmented with probabilities. States represent possible configurations of a system. Transitions among states occur at a discrete time and have an associated probability. DTMCs are discrete stochastic processes with the Markov property [4] [6], according to which the probability distribution of future states depend only upon the current state. Formally, a labeled DTMC is tuple $M=\left(S, S_{0}, P, L\right)$ :

- $S$ is a finite set of states 
- $S_{0} \in S$ is a set of initial states

- $P: S \times S \rightarrow[0,1]$ is a transition probability matrix where $\sum_{s^{\prime} \in S} P\left(s, s^{\prime}\right)=1$ for all $s \in S$.

- $L: S \rightarrow 2^{A P}$ is a labeling function which assigns to each state a set of Atomic Propositions which are true in that state.

A DTMC model is described as a number of modules which can interact with each other. After definition of local and global variables, the behavior of each module is described by a set of commands with the form: []guard-> prob $_{1}:$ update $_{1}+\ldots+\operatorname{prob}_{n}:$ update $_{n}$. The guard is a predicate over all the variables in the model. Each transition represents an update which the module can make if the guard is true. The update is assigned a probability.

\subsection{PCTL and Probabilistic Model Checking}

PCTL is a logic language inspired by CTL [6]. In place of the existential and universal quantification of CTL, PCTL provides the probabilistic operator $\wp_{\star}($.$) , where p \in[0,1]$ is probability bound and $\bowtie \in\{\leq,<, \geq,>\}$. PCTL is defined by the following syntax:

$\Phi::=$ true $|a| \Phi \wedge \Phi|\neg \Phi|$ ø৯p $(\Psi)$

$\Psi::=X \Phi|\Phi U \Phi| \Phi U^{\leq t} \Phi$

Formulas $\Phi$ are named state formulas and can be evaluated over a Boolean domain (true, false) in each state. Formulas $\Psi$ are named path formulas, and describe a pattern over the set of all possible paths originating in the state where they are evaluated. The satisfaction relation for a state $s$ in PCTL is:

$$
\begin{aligned}
& s \models \text { true } \\
& s \models a \text { iff } a \in L(s) \\
& s \models \neg \Phi \text { iff } s \not \models \Phi \\
& s \models \Phi_{1} \wedge \Phi_{2} \text { iff } s \models \Phi_{1} \text { and } s \models \Phi_{2} \\
& s \models \delta \bowtie p(\Psi) \text { iff } \operatorname{Pr}(s \models \psi) \bowtie p
\end{aligned}
$$

The satisfaction relation for a path formula with respect to a path $\pi$ originating in $s($ i.e., $\pi[0]=s)$ is defined as:

$$
\begin{aligned}
& \pi \models X \Phi \text { iff } \pi[1] \models \Phi \\
& \pi \models \Phi U \Psi \text { iff } \exists j \geq 0 .(\pi[j] \models \Psi \wedge(\forall k: 0 \leq k<j . \pi[k] \models \Phi)) \\
& \pi \models \Phi U \leq t \Psi \text { iff } \exists 0 \leq j \leq t .(\pi[j] \models \Psi \wedge(\forall k: 0 \leq k<j . \pi[k] \models \Phi))
\end{aligned}
$$

PCTL is an expressive language that allows many interesting reliability-related properties to be specified. The most important case is a reachability property. A reachability property states that a state where a certain characteristic property holds is eventually reached from a given initial state. Such state may represent a failure state, in which a transaction executed by the system modeled by the DTMC eventually terminates, or a success state.

There are also operators as $S$ and $R$, which are not in the syntax but are well defined in the PRISM for analysis [4][7]. The $S$ operator is used to reason about the steady-state behavior of a model. The $R$ operator supports the specification and analysis of properties based on costs and rewards. Probabilistic model checking is a decision problem: Given a finite Markov chain $M$, and PCTL state formula $\Phi$, determine whether $s \models_{M} \Phi$. The following shows several examples for the reliability requirements:

- $P_{\geq 0.001}(1 \leq s \leq 2 U s=3)$ : The probability that the system stays between states 1 and 2 until it reaches state 3 , is greater than 0.001

- $P_{\geq 0.001}\left(1 \leq s \leq 3 U^{\leq 5} s=4\right)$ : The probability that the system stays between states 1 and 3 until it reaches state 4 within 5 steps, is greater than 0.001 
- $S<0.05[$ size $/ \max >0.75]$ : The long-run probability of the value of size/max being more than $75 \%$, is less than 0.05

\section{The FUJIMI System}

This section introduces the principle and the resilience strategy of the FUJIMI system. The FUJIMI system is mainly used in game machine currently. The Non-stop property of game machine is enforced by the Japanese Government to avoid an argument between the players and the operator if a game machine freeze 2 . Before the explanation of the FUJIMI system, we distinguish three kinds of initial process:

- Cold start: The process initializes the hardware, operating system and the user application when the power is on. In general, the process consumes much time.

- Hot start: The system initializes the operating system and user application, without initial processing of the hardware. The process needs less time than cold start, but the intermediate results of the user application are lost.

- FUJIMI reset: The periodical reset process for the resilience strategy of FUJIMI system. The process needs less time than cold start and hot start, and the intermediate results of the user application can be preserved.

\subsection{System Principle}

For an embedded system which works in an environment without operators present but must work continually, such as a wind velocity meter in open country, keeping the system working continually is an important issue. However, it is not rare for microcomputers of embedded system to fall into failures such as stop, freeze, and lockup, which are caused by many problems including illegal instruction, bus errors, etc. For example, the instruction pointer may read a data area as an instruction and give the wrong results, or the synchronizations may deviate within the plurality sequencers. Noise, such as an abnormal electrical shock in the circuit of the semiconductor is one general reason for these problems. These problems may cause the system to enter into an incorrect status temporarily and this will bring serious effects to the system.

Beside well-known methods to recover the system such as watch-dog, power reset and redundant hardware, there are also many resilience strategies such as DRB (Distributed Recovery Blocks), NVP (Nversion programming) and NSCP (N self-checking Programming) [8]. However, there is no omnipotent solution for malfunction and freezing of the micro-computer. The MPU malfunction can be categorized as below (level is $_{\text {is }}$ normal status) [9]:

- level $_{0}$ : Running normally without any problems

- level $_{1}$ : Spurious Interrupt is caused

- level $_{2}$ : One byte contents of RAM is corrupted or I/O direction is changed

- level $_{3}$ : Many contents of RAM are destroyed

- level $_{4}$ : CPU execution is into the abnormal condition

- level 5 : A virtual SCR (Silicon Controlled Rectifier) is triggered and active the latch up [10]

\footnotetext{
${ }^{2}$ The operation of FUJIMI system has already been proven over 30 million game machines. Dragonchip Inc., in Hong Kong, for example, has made one series of FUJIMI MPU.
} 


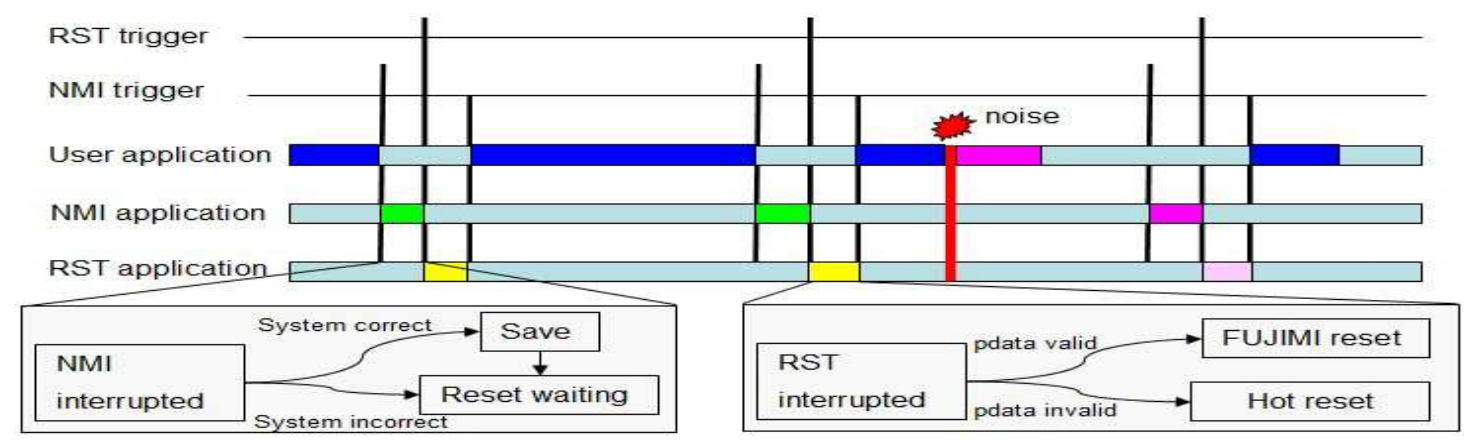

Figure 2: The System Principle

From level $_{0}$ to level $_{3}$, the errors can be fixed with the current technologies [3]. Once the violation condition on level $_{4}$ occurs, the MPU cannot resume by itself and must be rescued by a watch-dog in traditional systems. However, the watch-dog can rescue the embedded system only if the problems are detected. For level $_{5}$, there is no other way to escape from this except by using Power Off.

A resilience system named FUJIMI [9] is proposed to recover a system from temporary software failure at level $_{4}$. The FUJIMI system runs on a general purpose CPU such as M68030, which works between the hardware and the operating system. As shown is Figure 2 the clock periodically generates a Non-Maskable Interrupt $(N M I)$ and after some interval, generates a reset interrupt (RST) to the MPU. The intervals between two RST is called a cycle in this paper. When the FUJIMI system receives NMI, it saves backup data consisting of minimum information for recovery, and then enters into the waiting status. When the interrupt $R S T$ is signaled, the embedded system is reset with the valid backup data, or initializes a hot start when there is no valid backup data and the system is forced to execute from the beginning of an application. The FUJIMI resilience strategy has the following advantages:

- The strategy recovers systems much faster than general resilience strategies such as watch-dog, which is essential for time-response critical systems. Temporal system failures can be resumed by a short periodical cycle process.

- The applications can be restarted without loss of the intermediate results. The temporal failure caused by noise has no apparent effect on the application programs.

- The resilience strategy is implemented without redundancy hardware. This strategy is useful for a system that has limitation of power or cost.

\subsection{Resilience Strategy}

As previously discussed, the system uses two kinds of signal: NMI and RST. The basic idea lies in periodically backing-up the data with $N M I$ signal and recover with RST signal. We denote the current data as cdata, and denote the backup data as pdata. The data for recovery are saved $n$ times as pdata $_{1}$, pdata $_{2}, \ldots$, pdata $_{n}$. Each recovery data can be reused $m$ times, and keeps valid until it is used for $m$ times, where it becomes invalid which represents that it cannot be used anymore.

When NMI signal arises, the FUJIMI system checks whether $C P U$ is in the normal state by checking the program counter and the stack pointer. As in the upper part of Figure 3, if the $M P U$ is in the normal 


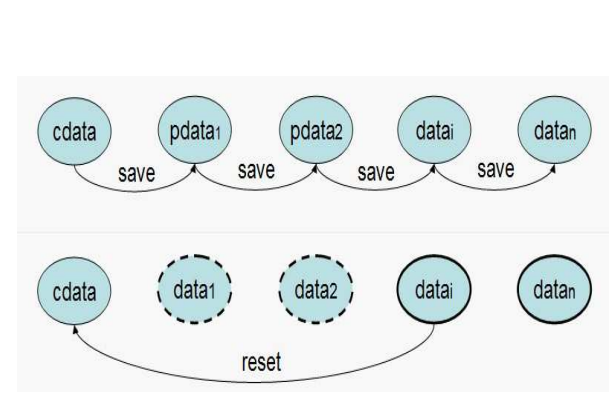

Figure 3: The Save and Reset Process

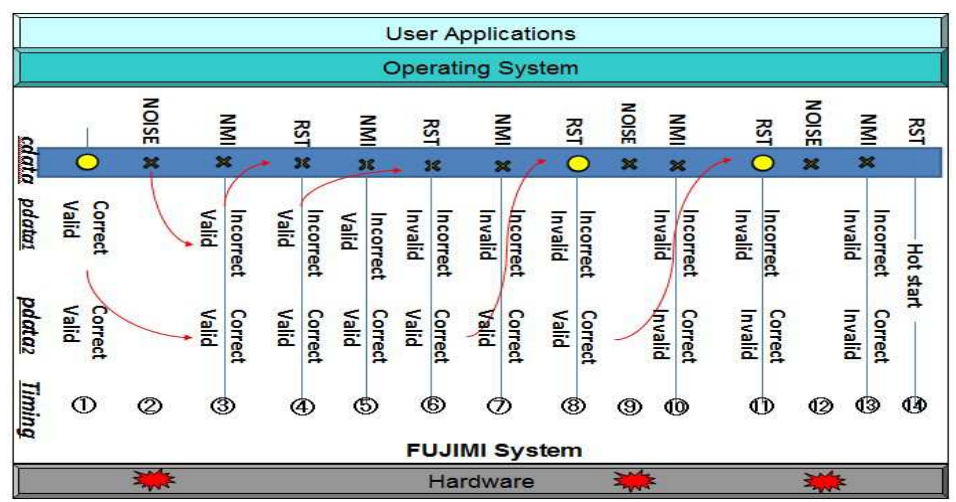

Figure 4: The Recover Process

state, the save process is carried out from right to left as pdata $a_{n-1}$ to pdata $_{n}$, pdata $a_{n-2}$ to pdata $_{n-1}$, $\ldots$, data $_{2}$ to pdata $_{1}$, and finally cdata to $p$ data $_{1}$. All the pdata are marked as valid and the initial number $m$ is assigned to the usage times. If the CPU is not in the normal state, the NMI interrupt is ignored and the system starts an undisturbed loop until an RST interrupt arises.

When the FUJIMI system receives RST signal, the reset exception handler checks the contents of RAM. If the FUJIMI system is not in the procedure for cold start or hot start, the most recent valid pdata is copied to $c d a t a$ as in the lower part of Figure 3 (the actual line represents that the data is valid and the dotted line represents invalid data). For example, if the pdata 1 is valid, then the CPU state is recovered from $p d a t a_{1}$, the usage time will becomes $m-1$. If $p d a t a_{1}$ has been used for $m$ time and became invalid, the system will try to recover from the pdata. The process is similar until pdata $a_{n}$ is exhausted and becomes invalid. When all the pdata are invalid, the system cannot be recovered and then the hot start begins. In this manner, this system achieves $m * n$ times of software redundancy without overage cost and power consumption caused by hardware redundancy.

Figure 4 shows an example of the recovery process with 2 previous data $p d a t a_{1}$ and $p d a t a_{2}$, and each pdata can be reused 2 times. The process recovers 2 noise effectiveness within 4 cycles. However, the system cannot be rescued when the $3^{\text {rd }}$ noise occurs, where both the two pdata are invalid, and then a hot start begins. The following steps from 1-14 explain the process of recovery. The number for the items corresponds to the timing shown with the underlining in Figure 4

1. After the cold start finishes normally, the initial system status in which the cdata, pdata $a_{1}$ and pdata $_{2}$ are correct, and both pdata $_{1}$ and pdata $a_{2}$ are valid.

2. Noise occurs for the $1^{\text {st }}$ time and makes the cdata incorrect.

3. NMI is triggered. The cdata is incorrect and detection fails; the correct pdata $a_{1}$ is saved to $p d a t a_{2}$, and the incorrect $c$ data is saved to pdata $a_{1}$.

4. The incorrect data $_{1}$ is reset to $c$ data, and the usage times decrease from 2 to 1 .

5. The incorrect cdata is detected, and the save process of $N M I$ fails.

6. The incorrect $p d a t a_{1}$ is reset to cdata, and then $p d a t a_{1}$ becomes invalid after decrementing by one for the second time.

7. The incorrect cdata is detected, and the save process of $N M I$ fails.

8. The correct $p d a t a_{2}$ is reset to cdata, and then the usage times decreases from 2 to 1 because pdat $_{1}$ is invalid. The system is recovered for the $1^{\text {st }}$ noise. 
9. Noise occurs again and makes the cdata incorrect.

10. The incorrect cdata is detected, and the save process of $N M I$ fails.

11. The correct pdat $_{2}$ is reset to $c$ data, and then becomes invalid after minus 1 for the $2^{\text {nd }}$ time. The system is recovered for the $2^{\text {nd }}$ noise.

12. Noise occurs $3^{\text {rd }}$ times and makes the cdata incorrect; no more valid pdata can be used for recovery because pdata $_{1}$ and pdata $_{2}$ are both invalid.

13. The incorrect cdata is detected, and the save process of $N M I$ fails.

14. The hot start process begins.

As previously noted, evaluating resilience against noise is another important theme in the reliability of a system. Until our work, implementation tests have been the most accurate evaluation method. However, these tests need a long monitor test for a large amount of samples. For example, for the software error rate of a product, intended to evaluate the ability of several hundred samples, requires 20,000 hours for testing [10].

\section{Probabilistic Model}

This section explains the modeling for the FUJIMI system described in Section 3, Our objective is to build a probabilistic model for evaluating resilience strategies against noise-attack behaviors. The model must simulate the resilience strategy, but not cause an explosion problem [6]. Furthermore, the model must be flexible for a variety of different configurations for system optimizations.

The model is constructed with Discrete Time Markov Chains (DTMCs) as introduced in Section 2.1 We comprehensively explore our model's multi-dimensional parameter space by systematically varying two key probabilistic parameters, including:

- The noise occurrence probability: The external environment electronic shock makes the cdata incorrect and makes the embedded system fall into failure. The probability value depends on the external environment where the embedded system works.

- The err detection probability: The detection of an error in the current data is also another probability, which means that an incorrect cdata will be either be detected or ignored. The probability value depends on the detection ability of the hardware.

The model is decomposed into three modules: Timer, Recovery Data, and Recover Function in Figure 5 The definition is in Figure 6 Recovery Data and Recover Function interact and coordinate with each other synchronizing with Timer. Actions are used to force two or more modules transition simultaneously [4]. For example, the following codes are from Timer module and Recovery Function module respectively. The transitions are synchronized with the action tick, which simulates the clock $t$ in the embedded system. The upper code refers that the noise occurs with the probability prob_noise when the clock ticks forward before reaching $t c$ (time cycle). The lower code refers that the CPU keeps normal before the clock reaches NMI if the noise does not occur.

$$
\begin{aligned}
& {[\text { tick }](t<t c) \rightarrow \text { prob_noise }:(\text { noise } I=\text { true }) \wedge(t \prime=t+1)+(1-\text { prob_noise }):(\text { noise }=\text { false }) \wedge(t \prime=t+1)} \\
& {[\text { tick }](t<N M I) \wedge(\text { cpu }=\text { normal }) \wedge(\text { noise }=\text { false }) \rightarrow(\text { cpul }=\text { normal })}
\end{aligned}
$$



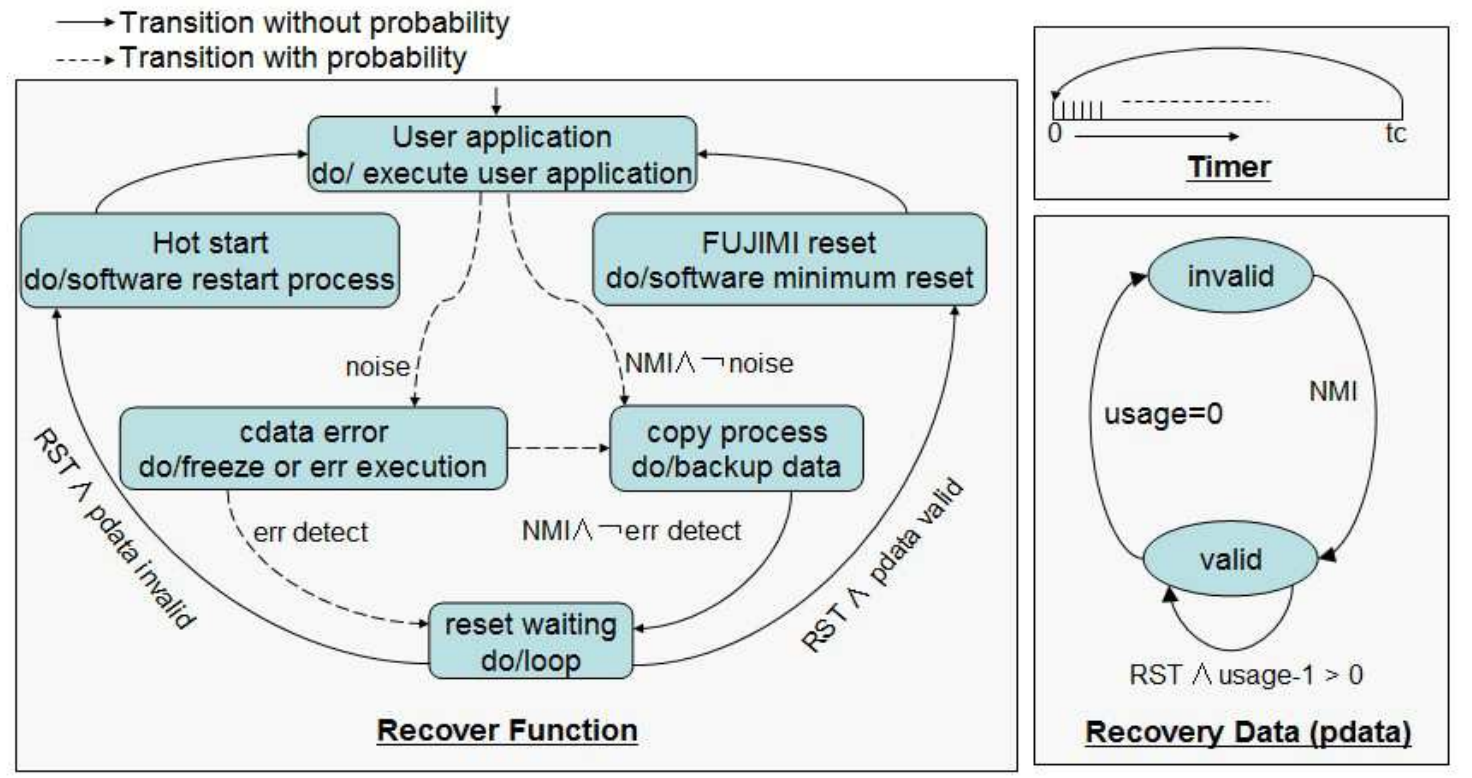

Figure 5: The Model Transitions

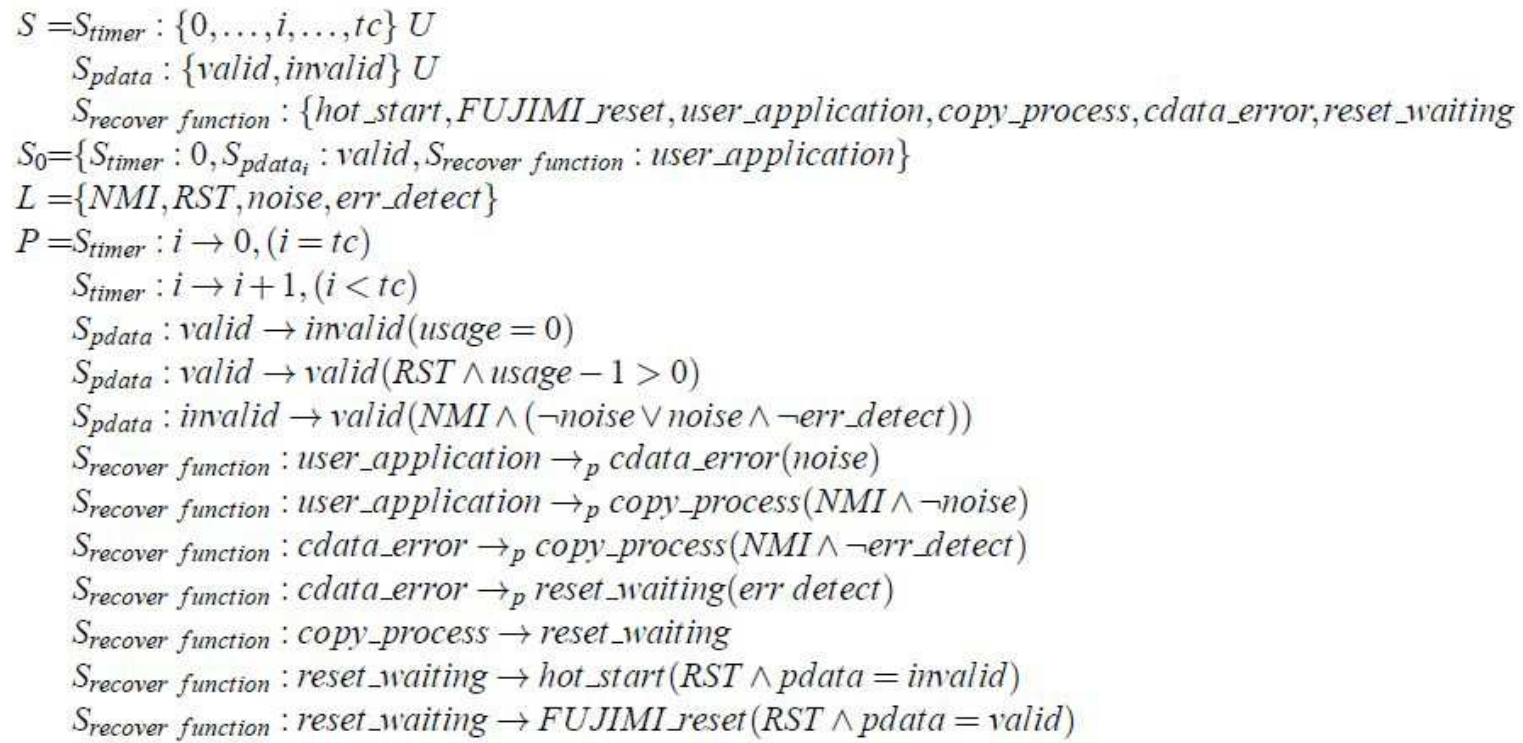

$\rightarrow_{p}$ : the transition with probability; $\rightarrow$ : the transition without probability.

* The transitions keep self-loop before the triggers occur, but the self-loop transitions are omitted in the graph.

Figure 6: The Model Definition 
Timer: The timer has a simple loop transition as $0 \rightarrow 1 \rightarrow 2 \cdots \rightarrow t c \rightarrow 0 \rightarrow 1 \rightarrow \ldots$ with an assigned cycle value $t c$. The noise occurs with a probability for each tick according to the environments. The transitions are shown in Figure 5(Timer).

Recovery Data (pdata): As shown in Figure 5 (Recovery Data), pdata transits between valid and invalid, according to the system status and recover strategy. The transitions are deterministic. Each pdata has a Boolean value that records if the data is correct, and an Integer value to record the usage times.

At the reset timing when RST signal arises, the pdatas transit self-loops with a decreasing usage number, and transit from valid to invalid when the pdata exhausts to 0 . At the save timing when NMI is triggered, the state transits from invalid to valid and the usage is set to the initial number $m$.

Recover Function: As shown in Figure 5(Recover Function), the recover function processes the recover algorithm for save and reset when $N M I$ and $R S T$ interrupts are triggered respectively, and also processes the corresponding process when noise occurs. The cdata and pdata are operated by the recover function, while synchronizing with the Timer. The initial state is user_application representing the process when the cold_start has finished correctly.

For the recover function, the system transits within the self-loop at the state user_application when the user application executes correctly. noise will occur with probability, which causes $c d a t a$ to transit to the status cdata_error. At the timing of NMI, if cdata is correct or if cdata is error, but is not detected, the system will enter into the copy_process status where the process of Figure 3 (upper part) is carried out. After copy_process finishes, the system will enter into reset_waiting. If the error status is detected, and the system will enter into reset_waiting immediately. In this case, the NMI will be ignored and the copy process in Figure 3 (upper part) will not be carried out.

The state transits with a self-loop at the state reset_waiting until the RST signal arises. At the timing where the RST exception occurs, the system will enter into the FUJIMI_reset where the process of Figure 3 (lower part) is carried out if there is a valid pdata. But when all the pdata become invalid and there is no valid data for system recovery, the system transits to hot_start state.

\section{Qualitative and Quantitative Evaluations}

PRISM supports the automated analysis of a wide range of qualitative and quantitative properties of probabilistic models with the probability temporal logic PCTL. In this section, we illustrate the qualitative and quantitative evaluations for the FUJIMI system, using the experimental data with three applications named Sensor, Logger and Ballon as shown in Table 1 . The experimental environment was as follows. Model checker: PRISM 4.0.3; CPU: Intel 1.70GHz; 2.9 GB RAM. The code for the model was about 130 lines.

NMI Process is the time for the NMI process to save pdata. RST Process is the time for FUJIMI process to reset pdata. NtoR Duration is the time from the beginning of periodical NMI to the beginning of the RST; User Application represents the continuous application operation time, which must not be disturbed. For example, if an application communicates with the MPU, time for the minimum necessary time must be reserved because it must not be disturbed. NtoR Duration is usually set to 3 times that

\footnotetext{
${ }^{3}$ Sensor receives the data from the anemometer and then sends the data to a processor; Logger saves the data received from the sensor to SD memory; Balloon is an action game with a touch panel. The periods of time are measured through the embedded codes. The embedded codes manipulate the external terminal, and the oscilloscope records the external terminals changes.
} 


\begin{tabular}{|l|c|c|c|c|c|c|}
\hline \hline & NMI Process & RST Process & NtoR Duration & User Application & Hot Start & Cold Start \\
\hline Sensor & $9.6 \mu \mathrm{s}$ & $16.2 \mu \mathrm{s}$ & $500 \mu \mathrm{s}$ & $14.9 \mathrm{~ms}$ & $110 \mu \mathrm{s}$ & $117 \mu \mathrm{s}$ \\
\hline Logger & $94 \mu \mathrm{s}$ & $114 \mu \mathrm{s}$ & $300 \mu \mathrm{s}$ & $49.6 \mathrm{~ms}$ & $170 \mathrm{~ms}$ & $220 \mathrm{~ms}$ \\
\hline Ballon & $66 \mu \mathrm{s}$ & $84 \mu \mathrm{s}$ & $25 \mu \mathrm{s}$ & $14.4 \mathrm{~ms}$ & $76 \mathrm{~ms}$ & $780 \mathrm{~ms}$ \\
\hline
\end{tabular}

Table 1: Configuration of 3 applications

of NMI Process time for a sufficient process. The periodical cycle must be larger than the total of NtoR Duration, RST Process and User Application. Hot Start is the process time to reset when there is no pdata to use. In this case, the system restarts from the beginning of the application. Cold Start is the process time for the system to recover from failure status without the FUJIMI system. We provide the following evaluations for the model:

- Qualitative verification:

- Formal proves: We formally prove that the system satisfies the desired properties.

- Quantitative assessment:

- System Configuration Optimization: We find the optimal value of the system's configurations according to different noise environments in which the embedded system works.

- System Failure Reduction: We give quantitative evidence to demonstrate that system failure is reduced.

- Average DownTime Hours/Year: ADT is an average value, which is the time to show how long the system is out of work for 1 year. ADT is the general industry criteria. From our analysis, the FUJIMI system decreases the ADT (improves the effectiveness) for most of real world applications.

\subsection{Qualitative Verification}

This process formally proves that the model complies with the resilience strategy requirements. Qualitative properties based on Markov chains typically require certain events with probability. These properties can be used to verify persistence and reachability [6]. We applied the following properties expressed with PCTL formulas $1-6$ (described below) to the model, and refined the model until the values of formula are satisfied 4 . The model is refined until the above formulas are satisfied, that demonstrates the model comply the desired properties.

(1) $: \mathbf{P}=?[\mathbf{F ~ G}($ cdata_error $)]$

(2) : $\mathbf{A}\left[\mathbf{G}\left(\right.\right.$ usage $_{\left(\text {pdata }_{i}\right)}<$ usage $\left._{\left(\text {pdat }_{j}\right)} \wedge(i<j)\right]$

(3) : $\mathbf{P}^{\geq 1}[\mathbf{F}$ hot_start $]$

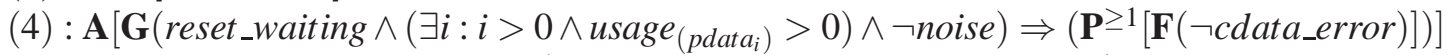

$(5): \mathbf{A}\left[\mathbf{G}\left(\right.\right.$ noise $\wedge$ reset_waiting $\wedge\left(\nexists i: i>0 \wedge\right.$ usage $\left.\left._{\left(\text {pdat }_{i}\right)}>0\right)\right) \Rightarrow(\mathbf{P} \geq 1[\mathbf{F}($ hot_start $\left.)])\right]$

(6) $: \mathbf{S}=$ ? [hot_start $]) /(\mathbf{S}=$ ? $[$ cdata_error $] \leq 1$

Formula (1) is used to inquire the probability that cdata can always be wrong in the future. This value must be 0 for the following reasons: The system can be recovered with pdata if there is a valid pdata, and can be reset initially if all the pdata are invalid. The algorithm does not allow the wrong

\footnotetext{
${ }^{4}$ The formulas are expressed in this paper for convenience, but may be different with actual PCTL formulas. For example, $\exists i \wedge$ usage $\left(\right.$ pdata $\left._{i}\right)>0$ is usage $_{\left(\text {pdata }_{1}\right)}>0 \vee$ usage $_{\left(\text {pdata }_{2}\right)}>0$ if there are two pdata in the model because the operators as ' $\exists$ ' are illegal in DTMC.
} 
status of cdata to continue forever. Formula (2) shows that the process for $R S T$ is always from the most recent valid pdata (usage $_{(\text {pdata) }}$ is the available usage number of pdata). This number means that on All the path, the Global samples satisfy that the pdata with smaller subscript is always used before the larger subscript.

Formula (3) means that no matter how small the probability, the hot_start status can be reached. Formula (4) can be read as: when the system enters into the loop of reset_waiting status, and there is a valid pdata, the system can be recovered if the noise no longer occurs. This property is itself resilient and can be understood easily. Formula (5) means that if there is no valid data for recovery and the noise occurs, the system will definitely enter into hot_start.

Let $\mathbf{S}=$ ? [cdata_error $]$ and $\mathbf{S}=$ ? [hot_start $]$ be the values associated with the average of the cdata_error statistic and hot_start statistic. Formula (6) implies the probability that hot_start is always smaller than the probability of cdata_error caused by noise. This Formula verifies that the FUJIMI system is always effective in reducing system failure.

\subsection{Quantitative Assessment}

This section provides three kinds of quantitative assessment: System configuration optimization, system failure reduction, and Average DownTime.

\subsubsection{System Optimizations}

In this section three kinds of configurations are adjusted to achieve the minimum system failure or the highest effectiveness.

Usage Times for pdata: We change the usage times of pdata from 1-3 and the results are shown in Figure 7 The horizontal axis is the increasing probability of noise occurrence (with a base of 10,000, for example 57.5 on the horizontal axis represents the noise probability of 57.5/10,000), and the vertical axis is the probability of system failure. The result shows that the higher the usage time, the lower the probability of system failure. The difference increases as the noise occurrence decreases. The values can help users to properly select usage times for different noise environments and the user's requirements of system failure probability.
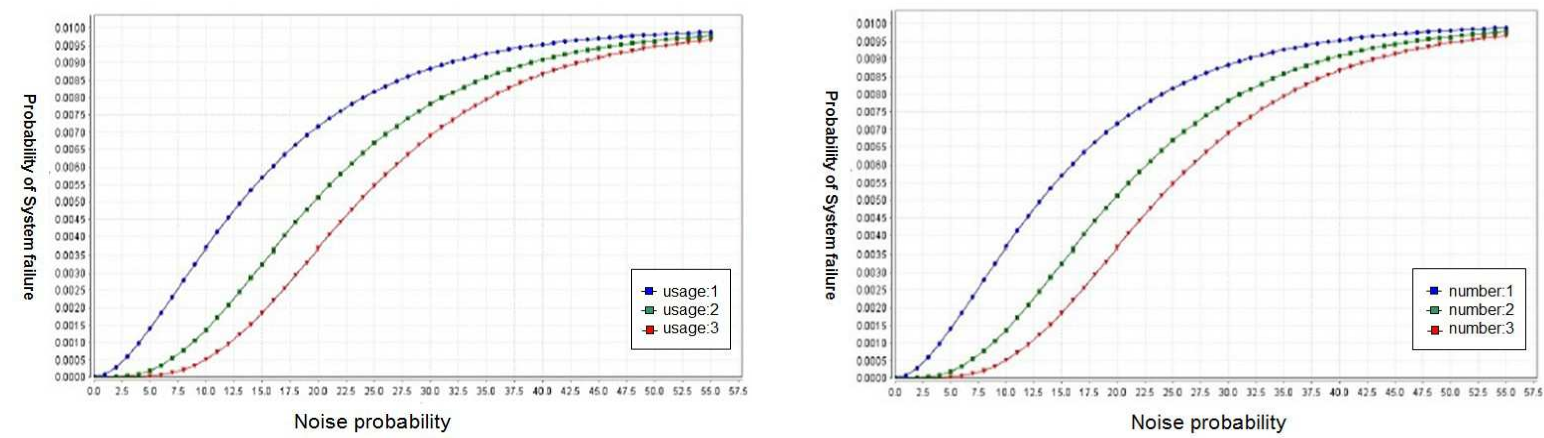

Figure 7: The System Failure Probability for Different Usage Figure 8: The System Failure For Different Recovery Data Times Numbers

The Recovery Data Number: Another system quantity which can be optimized is the pdata number. The number can be adjusted to achieve the most reduction in system failure. The probability of system 
failure decreases when the recovery data number increases. In the experiments, we sample the $\mathbf{S}=$ ?[hot_start] for different noise probabilities. The result is shown in Figure 8 The horizontal axis is the increasing probability of noise occurrence (with a base of 10,000, for example 57.5 on the horizontal axis represents the noise probability of 57.5/10,000), and the vertical axis is the probability of system failure. The system failure is reduced as the number increases, but a higher number implies memory consumption and a higher cost in time for the copying process.

Periodical Cycle: For the resilience strategy described in Section 3 , when the noise causes an error, the system runs into a waiting state until the reset signal RST arises. In different environments, noise will occur frequently or sparsely. If the noise occurs frequently, then the period must be shorter, which consequently, makes the waiting time shorter. On the contrary, if the noise occurs sparsely, the periodical cycle must be longer to reduce the overhead of the save and reset process.

An optimized cycle can be found with the evaluation of system effectiveness. For this goal, after every kind of transition is annexed with a cost (called reward in PRISM), the proportion of the execution time for user application to all the elapsed time is calculated. Formula (7) is the proportion of time of user application to all the elapsed time representing the effectiveness of FUJIMI system. The value in Formula (7) is annexed with the user application Balloon in Table 1 As the formula is to calculate the proportion of weight available $_{\text {and weight }}$ total , the values in Formula (7) are 10 times smaller than the actual values in Table 1 to avoid the explosion problem. Timer costs 1 for user application and reset waiting, but costs 7,600 for hot start for each tick.

$$
\text { (7)Effectiveness }=\mathbf{R}\left\{\text { “weight } \text { available }_{\text {weight }} \text { total" }\right\}
$$

\begin{tabular}{l|c} 
rewards weight total & rewards weight available $_{\text {user_application }: 1 ;}$ \\
reset_waiting $: 1 ;$ & user_application : $1 ;$ \\
hot_start $: 7,600 ;$ & endrewards \\
endrewards &
\end{tabular}
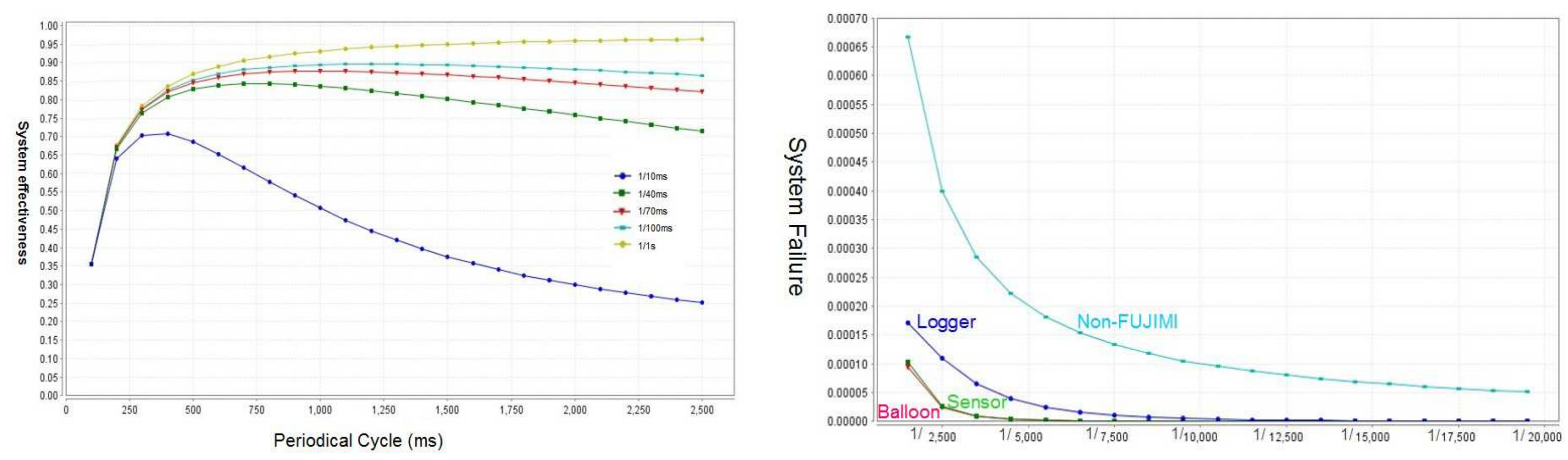

Figure 9: The System Effectiveness for Different Cycle Figure 10: The System Failure with and without FUJIMI Period System

Figure 9 shows that the most effective time cycles are different for different probabilities of noise. The horizontal axis is the value for the cycles. The 5 lines represent the effectiveness for 5 kinds of noise probability of $1 / 10 \mathrm{~ms}, 1 / 40 \mathrm{~ms}, 1 / 70 \mathrm{~ms}, 1 / 100 \mathrm{~ms}$ to $1 / 1 \mathrm{~s}$. The most effective point of the cycle period can be found from the graph. For example, the best effective point is $4 m s, 9 m s, 12 m s, 14 m s, 25 m s$ for the 5 kinds of noise probability respectively. The results show that the periodical cycle should be set shorter 
if the overhead is small enough because this can avoid the reset waiting to consume much of the effect time. However, when the noise probability is small, the values increase with a slow curve after violently bending points. As mentioned at the beginning of this Section, the cycle must be larger than the total value of User Application time, NtoR Duration time, and RSR Process time. The optimized cycle must be set to the larger one between the total value and the bending point.

\subsubsection{System Failure Reduction}

After optimization, the system becomes deterministic, and the system failure with and without FUJIMI system can be calculated respectively.

(8) : Failure $=\mathbf{S}[$ hot_start $]$

The results of Figure 10 serve to demonstrate that the FUJIMI system reduces system failure caused by noise. The horizontal axis represents the decreasing noise probability and the vertical axis represents the statistics value of the times that the system enters into Hot Start states as shown in Formula (8). The most upper line represents the probability of system failure without the FUJIMI system, and the lower three lines represent the system failure of the three applications in Table 1 with the FUJIMI system.

\subsubsection{Average DownTime (ADT)}

Average DownTime (ADT) stands for an industry a criterion that evaluates systems which must run continually. The value is the average time in which systems malfunction and cannot work within one year. We use formula (9) to evaluate the ADT. 8,760 is the hours in one year. weight $t_{\text {total }}$ represents the total process time, and weight available $_{\text {is }}$ the effective process time for user application. Cost for hot_start is substituted as 11, 17,000, 7,600 for the user application Sensor, Logger, Ballon respectively (10 times smaller than the actual values).

(9)ADT $=8760 *\left(1-\mathbf{R}\left\{\right.\right.$ “weight ${ }_{\text {available }} /$ weight $_{\text {totol }}$ " $\left.\}\right)$

\begin{tabular}{l|c} 
rewards weight $t_{\text {total }}$ & rewards weight available \\
user_application $: 1 ;$ & user_application $: 1 ;$ \\
reset_waiting $: 1 ;$ & endrewards \\
hot_start $: 7,600 ;$ & \\
endrewards &
\end{tabular}

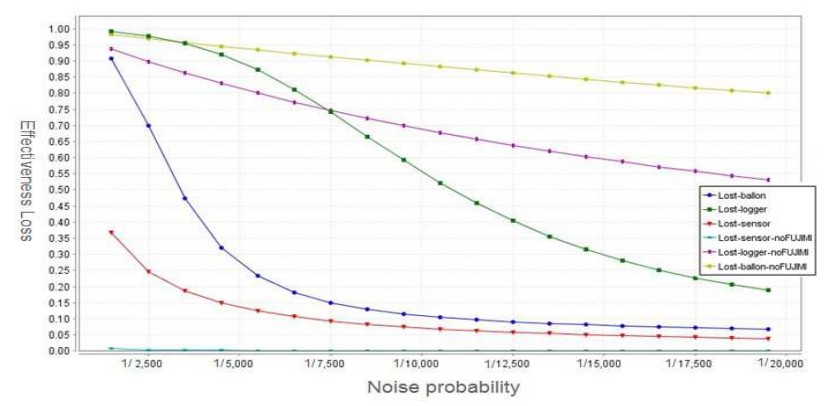

Figure 11: Effectiveness Loss With and Without FUJIMI
Figure 11 shows three pairs of lines for the applications in Table 1 Each pair indicates the values of loss proportion (1$\mathbf{R}\left\{\right.$ "weight available $_{\text {weight }}$ totol" $\}$ ) with and without the FUJIMI system when the noise probability decreases along the horizontal axis. The restart processes without FUJIMI consume the Cold Start time in Table 1

The application Sensor has a light process time for the Cold Start process. The FUJIMI system makes the effectiveness worse because of the waiting time as well as the overhead in each cycle. For the application Logger, because the 
overhead and Hot Start time are relatively heavy, the FUJIMI system is beneficial from the crossing point where the noise probability is $1 / 75,000 \mu \mathrm{s}$. In other words, the FUJIMI system improves the system effectiveness only if the noise probability is smaller than $1 / 75,000 \mu \mathrm{s}$. In the real world, the noise occurs with far smaller probability than $1 / 75,000 \mu \mathrm{s}$. For the applications Ballon, the FUJIMI system brings absolute benefit as the overhead and restart time are heavy.

The results compare the effectiveness loss with and without FUJIMI system, and indicate that the FUJIMI system makes the effectiveness worse if the process for Cold Start is light enough. Nonetheless, the system without FUJIMI system is an ideal situation in that whenever the error status occurs the restart can be processed immediately, which is impossible in the real world. In general, the processes are delayed only after resilience functions as the watch-dog or manual power reset. This latency cannot be predicated. As the applications represent typical characters with the light, middle and heavy Cold Start processes, we can affirm that the FUJIMI system can bring benefit to most real world systems.

\section{Related Work}

Few works have provided an analysis for temporary system failure caused by noise, which, in real world, cannot be ignored. Our target system is very original for this kind of temporary system failure, which overall improves resilience with minimum software redundancy. Although there are not exactly similar related works, several works for resilience systems or fault tolerance with probabilistic model checking exist.

The paper [8] presented a quantitative analysis to three kinds of resilience models using the Molkov reword model. This work can be considered as an elementary prototype of the kind of work described in this paper, but is different from our work, in that our target resilience system is very light $\mathrm{N}$-version programming. There is no redundancy hardware, and the software alternative needs only minimum recovery data for a temporary effect from noise.

Another paper [11] presented an approach for the verification of self-adaptive systems that relies on probabilistic model-checking for obtaining levels of confidence regarding trustworthy service delivery when a system undergoes adaptation. An environment is stimulated to trigger system adaptation mechanisms, to collect experimental data, to trace the system undergoing adaptation, and to generate a model from the trace, and then verify the system properties. This work uses a quantitative assessment for a self-adaptive algorithm. Our work also uses a quantitative assessment, but our work provides more and different evaluations. Moreover, the model is initially constructed before evaluations in our work.

Still another paper [12] presented a simulation prototype for experimenting with resilience strategies for network systems. This work supported expectations about resilience strategies and revealed strategy assumptions, unexpected emergent situations, and insights into strategy configurations. Evaluation of the system configurations to achieve optimized effectiveness is also a part of our work. But our model is different because both the evaluation object and methods are different. We formally model a light-weight redundant system with software against temporary system failure caused by noise, and to provide formal qualitative and quantitative evaluations.

\section{Summary of the Contribution and Future Work}

This paper presented qualitative and quantitative evaluations for a novelty resilience system named FUJIMI. The resilience system reduces temporary software failure caused by external noise with a lightweight data-backup mechanism. Until our work, implementation testing has been the most used method 
to evaluate system failure by the software error, but this method is time consuming and costly and only provides a low accuracy. Our method solved the difficult problem of evaluation for such kind of systems without a large testing sample. The formal results from the formal model were accurate and reliable.

The qualitative evaluations verified that the FUJIMI system complies with resilience strategy, and the quantitative evaluations provided assessments of the FUJIMI system such as failure reduction and ADT. Moreover, the system configurations were optimized. Our method has been demonstrated to be very effective through three real world applications. Our systematic evaluation methods for qualitative and quantitative evaluations can be extended to other systems that have probabilistic behaviors.

However, the evaluations only include temporary system failure on level $_{4}$. Safety Integrity Level (SIL) [13] is widely used to define the safety properties of software. In the future, we plan to evaluate the FUJIMI system including the system failure from level $_{0}$ to level $_{5}$, and provide assessment values for IEC 61508 [13].

\section{Acknowledgments}

The authors would like to thank Isao Tatsuno of LETech Co., Ltd and Junzo Kenematu and Hajime Shirai of System Consultants Co., Ltd, who greatly contributed to this research.

\section{References}

[1] N. Kanekawa, E. Ibe, T. Suga, Y. Uematsu: Dependability in Electronic Systems: Mitigation of Hardware Failures, Soft Errors, and Electro-Magnetic Disturbances, ISBN-10: 1441967141, 2010.

[2] C. G. Rieger, D.I. Gertman: Resilient Control Systems: Next Generation Design Research. IEEE Conference on Human System Interaction.doi:10.1109/HSI . 2009.5091051.

[3] Y. Joseph: The Definitive Guide to the ARM Cortex-M3, Second Edition. ISBN-13: 978-1856179638, 2009.

[4] Official website: http://www.prismmodelchecker.org.

[5] A. Hinton, M. Kwiatkowska, G. Norman, and D. Parker. PRISM: A tool for automatic verification of probabilistic systems. Proc. 12th International Conference on Tools and Algorithms for the Construction and Analysis of Systems, volume 3920 of LNCS, pp. 441-444. Springer, doi:10.1007/11691372_29, 2006.

[6] C. Baier and J.P. Katoen. Principles of Model Checking. MIT Press, ISBN-13: 978-0262026499, 2008.

[7] W. J. Stewart. Introduction to the Numerical Solution of Markov Chains. Princeton, ISBN-10: 0691036993, 1994.

[8] B. D. Joanne, A. D. Stacy, F. A. Patterson-Hine. Simple Models of Hardware and Software Fault Tolerance, Proc. Annual Reliability and Maintainability Symposium, doi:10.1109/RAMS .1994.291094.

[9] FUJIMI Official website: http://www.fujimi.org.

[10] Reliability handbook(in Japanese): http://documentation.renesas.com/doc/products/others/r51zz0001jj0100.pdf, 2008.

[11] C. Javier, L. Rogerio. Evaluation of Resilience in Self-Adaptive Systems Using Probabilistic ModelChecking. Software Engineering for Adaptive and Self-Managing Systems (SEAMS), doi:10.1109/SEAMS . 2012.6224391.

[12] D. Lam, E. Skiles and P. Grisham. Simulation Tool for Evaluation and Design of Resilience Strategies. 6th International Symposium on Resilient Control Systems, doi:10.1109/ISRCS.2013.6623774.

[13] D. Smith, K. Simpson. Safety Critical Systems Handbook - A Straightforward Guide to Functional Safety, IEC 61508 and Related Standards 3rd Edition, ISBN 978-0-08-096781-3. 